\title{
Evaluation of the Verigene ${ }^{\circledR}$ Blood Culture Nucleic Acid test for rapid identification of gram positive pathogens from positive blood cultures
}

\author{
Agnese Cellini, Maria Federica Pedna, Francesca Del Bianco, Vittorio Sambri \\ Unit of Microbiology, The Greater Romagna Hub Laboratory, Pievesestina di Cesena, Italy
}

\section{Summary}

Background. The rapid identification of the etiology and the evaluation of the antimicrobial susceptibility of the bacteria causing bacteremia is of outmost relevance to set up an adequate treatment of sepsis. In this study we evaluated the microarray based method, Verigene Gram-positive blood cultures (BC-GP) nucleic acid test (Nanosphere Inc., Northbrook, IL, USA) for the identification of Gram positive pathogens from positive blood cultures. The panel BC-GP is capable to identify 13 germs and 3 genes associated with antimicrobial resistance.

Materials and Methods. In this study a total of 100 positive, non replicated and monomicrobic blood cultures have been evaluated. For testing on the Verigene platform using the BC-GP assay, $350 \mathrm{~L}$ of blood culture media from a positive the blood culture bottle.

Results. A total of 100 positive blood cultures were tested by the Verigene BC-GP assay: out of these a total of 100 Gram-positive cocci were identified. The most frequent bacteria identified included staphylococci, streptococci and enterococci. Among staphylococci, Staphylococcus aureus accounted for 25\% (15/60), with 38\% of S. epidermidis 37\% (23/60) and 37\% (22/60) other CoNS. All the $S$. aureus isolates were correctly identified by BC-GP whereas in $2 / 45$ cases (4\%)

Correspondence: Agnese Cellini, Unit of Microbiology, The Greater Romagna Hub Laboratory, P.le Liberazione 60, Pievesestina di Cesena 47522 (FC), Italy. Tel.: +39.0547 .394872 - Fax: +39.0547 .394865 .

E-mail: a.cellini@outlook.it

Key words: Sepsis; blood culture; Gram positive; rapid identification.

Acknowledgements: authors thank Nanosphere for kindly providing in the kits used in this study.

Contributions: the authors contributed equally.

Conflict of interest: the authors declare no potential conflict of interest.

Received for publication: 9 January 2015.

Accepted for publication: 29 May 2015.

(O) Copyright A. Cellini et al., 2015

Licensee PAGEPress, Italy

Microbiologia Medica 2015; 30:4945

doi:10.4081/mm.2015.4945

This article is distributed under the terms of the Creative Commons Attribution Noncommercial License (by-nc 3.0) which permits any noncommercial use, distribution, and reproduction in any medium, provided the original author(s) and source are credited.
BC-GP misidentified CoNS. In the case of enterococci $7 / 10$ were $E$. faecalis and $3 \mathrm{E}$. faecium, all of these were correctly identified.

Conclusions. The overall agreement with the results obtained by standard procedure is quite elevated (88\%) and as a consequence the BC-GP panel could be used as a rapid diagnostic tool to give a faster response in the case of bacteremia associated with sepsis.

\section{Introduction}

Sepsis, often a consequence of bacteremic infections, is a major cause of mortality worldwide. The rapid identification of the etiology and the evaluation of the antimicrobial susceptibility of the bacteria causing bacteremia is of outmost relevance to set up an adequate treatment of sepsis. The risk of death as a consequence of sepsis increases by $6-10 \%$ per hour in the absence of a pathogen effective and targeted therapy and it is well known that during the course of sepsis the efficacy and rapidity of specific treatment are greatly impacting on mortality ratio $(6,7,13)$.

The most commonly isolated bacteria from bloodstream infections belong to Gram positive: in particular many of the isolated strains are coagulase-negative staphylococci (CoNS) that are generally considered as contaminant organisms of little clinical significance $(3,10)$. Blood culture still are the gold standard diagnostic approach for the diagnosis of bacteremia related to sepsis (6).

The common procedure to identify a pathogen from a positive blood culture bottle include preparation of a Gram stained smear and the subsequent isolation of the germs by growing onto solid media, then followed by final identification by biochemical tools. A first empirical indication about the germ found is consequently made available within few minutes following the observation of the slide: this is generally communicated in order to allow an early set up of an empirical treatment $(1,2)$.

A precise identification and a complete evaluation of the antimicrobial susceptibility (AS) of the isolated strain is generally achievable after 18 to $48 \mathrm{~h}$ of incubation followed by a further $12-24 \mathrm{~h}$ period that is necessary for the identification and AS testing. This delayed answer is very often of limited impact onto the management of septic patients and as a consequence many efforts have been put in place to identify new techniques that can reduce the time to the final and complete report.

Among these methods, are included fluorescent in situ hybridization (FISH) and Matrix Assisted Laser Desorption Ionization Time-of-Flight (MALDI-TOF) $(5,8,12)$. These methods, however, are of limited utility when AS must be evaluated or in the case of polymicrobial infections. The use of genetic microarrays can somehow overcome many of the limitations of either FISH or MALDTTOF, since by using this technology pathogens are identified within a short time and quite a large panel of the genes associated with drug-resistance is generally reported $(4,11)$.

In this study, we evaluated the microarray based method, Verigene Gram-Positive Blood Cultures (BC-GP) nucleic acid test (Nanosphere 
Inc., Northbrook, IL, USA) for the identification of Gram positive pathogens from positive blood cultures.

The system uses oligonucleotide probes associated with gold nanoparticles for the automatic detection of molecular targets by hybridization to genomic sequences species-specific and genetic determinants of antibiotic resistance. Specifically, the panel BC-GP is capable to identify, from a primary volume of $350 \mathrm{~L}$ of positive blood culture the following germs: Staphylococcus spp., Streptococcus spp., Listeria spp., Micrococcus spp., Staphylococcus aureus, Staphylococcus epidermidis, Staphylococcus lugdunensis, Streptococcus pneumoniae, Streptococcus pyogenes, Streptococcus agalactiae, Streptococcus anginosus group, Enterococcus faecalis, Enterococcus faecium.

In addition the presence of the following genes associated with antimicrobial resistance is also made possible: mecA, vanA and vanB, All the procedures get the final end within a maximum time of $2 \mathrm{~h} 30$ minutes.

\section{Materials and Methods}

In this study, a total of 100 positive non replicated and monomicrobic blood cultures have been evaluated. The method used for the blood culture was the standard procedure based onto the BacTAlert system (Biomerieux, Marcy L'Etoile, France). A first evaluation of the germs performed by Gram staining of a smear was used to discriminate Gram positive from Gram negative bacteria: only the Gram positive were included into the study.

The Verigene platform includes the Verigene Processor $S P$ and Verigene Reader. The Verigene Processor SP carries out extraction of nucleic acid from specimens using magnetic glass beads.

Patient samples are loaded into an extraction tray, which is then loaded into the processor along with the utility tray, pipette tip holder assembly, and test cartridge. These items are all single-use disposable components that contain all the reagents required for testing. The Verigene Reader controls the processor and is responsible for specimen tracking, test selection, imaging, and analysis of test cartridges and display of the results. For testing on the Verigene platform using the BC-GP assay, $350 \mathrm{~L}$ of blood culture media from the positive aerobic bottle is loaded into the extraction tray, which is then placed into the processor $S P$ along with all other consumables. The instrument extracts nucleic acid from the sample which is then mixed with the appropriate buffer and transferred to the test cartridge. The target analyze, if present, hybridizes to synthetic gene-specific oligonucleotide capture strands on the test cartridge substrate slide. Another synthetic mediator target-specific nucleotide is introduced to form a hybridization sandwich with the gene of interest.

At this point, a gold nanoparticle-labeled probe is introduced with oligonucleotides complementary to the intermediate oligonucleotide bound to the gene of interest. Finally, the gold nanoparticles are coated with silver to enhance the optical signal. The test cartridge is then removed from the Processor $S P$, and the substrate slide is inserted into the Verigene Reader for analysis. The Verigene Reader projects white light across the substrate slide, detects the relative brightness of each spot due to gold nanoparticles bound to target-specific probes and provides a Detected or Not Detected result for each of the panel members. The results have been compared with those obtained by standard techniques and the discrepancies were basically investigated by $16 \mathrm{~S}$ rRNA sequencing after standard PCR amplification (9).

\section{Results}

A total of 100 positive blood cultures were tested by the Verigene BCGP assay: out of these a total of 100 Gram-positive cocci were identified. Of these, $8 \%$ (8/100) were found as polymicrobial by routine laboratory investigations. $97 \%$ (97/100) of the germs were included in the BC-GP panel, being only 3 out of the identifiable panel.

Table 1 summarizes the results. In detail, the most frequent bacteria identified included staphylococci, streptococci and enterococci

Table 1. Summary of the results obtained by Gram-positive blood cultures assay.

\begin{tabular}{|c|c|c|c|c|}
\hline Microrganism & Total isolates, $\mathbf{n}$. & $\begin{array}{l}\text { Isolates correctly } \\
\text { identified, n (\%) }\end{array}$ & $\begin{array}{c}\text { Isolates not detected, } \\
\text { n. (\%) }\end{array}$ & $\begin{array}{l}\text { Isolates incorrectly } \\
\text { identified, n (\%) }\end{array}$ \\
\hline $\begin{array}{l}\text { Staphylococcus aureus } \\
\text { MSSA* } \\
\text { MRSA** }^{*}\end{array}$ & $\begin{array}{l}15 \\
10 \\
5\end{array}$ & $\begin{array}{l}15(100) \\
10(100) \\
5(100)\end{array}$ & & \\
\hline $\begin{array}{l}\text { CoNS*** } \\
\text { S. epidermidis } \\
\text { Other CoNS }\end{array}$ & $\begin{array}{l}45 \\
23 \\
22\end{array}$ & $\begin{array}{l}43(96) \\
22(96) \\
21(95)\end{array}$ & & $\begin{array}{l}2(4) \\
1(4) \\
1(5)\end{array}$ \\
\hline $\begin{array}{l}\text { Enterococci } \\
\text { Enterococcus faecalis } \\
\text { Enterococcus faecium }\end{array}$ & $\begin{array}{l}10 \\
7 \\
3\end{array}$ & $\begin{array}{l}10(100) \\
7(100) \\
3(100)\end{array}$ & & \\
\hline $\begin{array}{l}\text { Streptococci } \\
\text { Streptococcus pneumoniae } \\
\text { Streptococcus pyogenes } \\
\text { Streptococcus agalactiae } \\
\text { Streptococcus anginosus group } \\
\text { Other streptococci }\end{array}$ & $\begin{array}{l}21 \\
5 \\
1 \\
2 \\
5 \\
8\end{array}$ & $\begin{array}{l}18(86) \\
5(100) \\
1(100) \\
2(100) \\
5(100) \\
5(63)\end{array}$ & & 3 (14) \\
\hline Total monomicrobial isolates & 91 & & & \\
\hline Other $^{\mathrm{a}}$ & 3 & & $3(100)$ & \\
\hline Other ${ }^{b}$ & 2 & & $2(100)$ & \\
\hline Lack of positive control & 4 & & $4(100)$ & \\
\hline Total number of isolates & 100 & & & \\
\hline
\end{tabular}




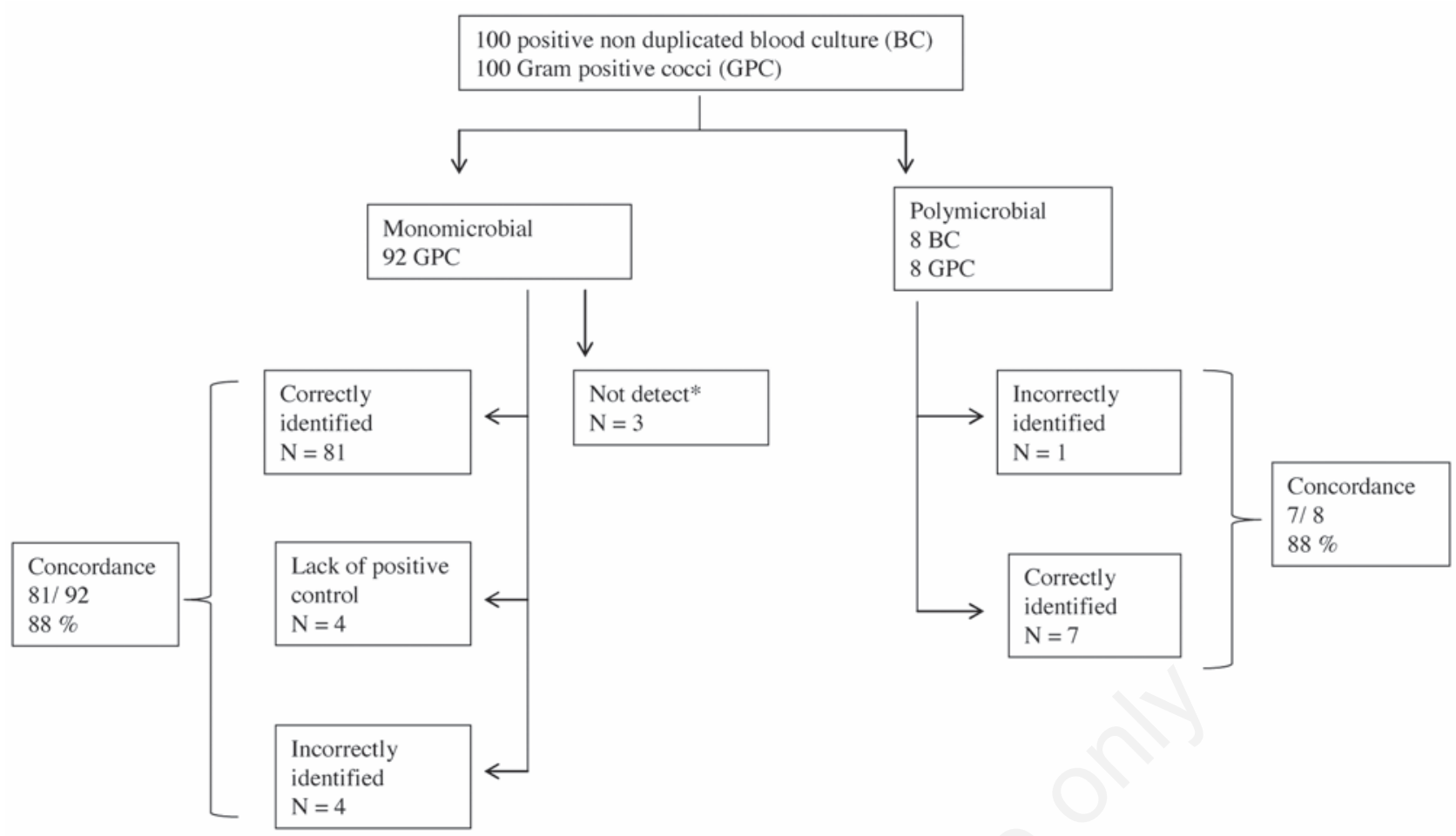

* Not in the panel

Figure 1. Performance of the Gram-positive blood cultures test for the identification of Gram-positive cocci from positive blood cultures.

with $60 \%, 21 \%$ and $10 \%$ of germs isolated, respectively. Among staphylococci, $S$. aureus accounted for $25 \%$ (15/60), with $38 \%$ of $S$. epidermidis $37 \%$ (23/60) and $37 \%$ (22/60) other CoNS. All the $S$. aureus isolates were correctly identified by BC-GP whereas in $2 / 45$ cases (4\%) BC-GP misidentified CoNS. In the case of enterococci $7 / 10$ were $E$. faecalis and $3 \mathrm{E}$. faecium, all of these were correctly identified by the Verigene test.

Three isolates (14\%) out of the 21 streptococci was misidentified by BC-GP. In total $88 \%$ of the bacterial isolates gave a concordant identification between standard colture and BC-GP. The discrepant results were generated by 3 germs that are not included in the BC-GP panel, $3 \%$ gave a non-compliant result, and in 4 case no result was generated due to instrumental failure (Figure 1).

\section{Conclusions}

The Verigene BC-GP assay was easy and rapid to be performed, The list of the identifiable germs is wide and covers about $85 \%$ of the pathogens identified in 2013 from positive blood cultures in the Unit of Microbiology, the Greater Romagna Hub Laboratory (Italy). The overall agreement with the results obtained by standard procedure is quite elevated (88\%) and as a consequence the BC-GP panel could be used as a rapid diagnostic tool to give a faster response in the case of bacteriemia associated with sepsis.

In addition, the capability of this method to discriminate between CoNS and other clinically relevant Gram positive species could be important to de-escalate the antimicrobial therapy when the presence of contaminants is highly likely $(3,10)$.

\section{References}

1. Barenfanger J, Graham DR, Kolluri L, et al. Decreased mortality associated with prompt Gram staining of blood cultures. Am J Clin Pathol 2008; 130: 870-6.

2. Beekmann SE, Diekema DJ, Chapin KC, Doern GV. Effects of rapid detection of bloodstream infections on length of hospitalization and hospital charges. J Clin Microbiol 2003; 41: 3119-25.

3. Beekmann SE, Diekema DJ, Doern GV. Determining the clinical significance of coagulase-negative staphylococci isolated from blood cultures. Infect Control Hosp Epidemiol 2005; 26: 559-66.

4. Buchan BW, Ginocchio CC, Manii R, et al. Multiplex identification of gram-positive bacteria and resistance determinants directly from positive blood culture broths: evaluation of an automated microarray-based nucleic acid test. PLoS Med 2013; 10: e1001478.

5. Deck MK, Anderson ES, Buckner RJ, et al. Multicenter evaluation of the Staphylococcus QuickFISH method for simultaneous identification of Staphylococcus aureus and coagulasenegative staphylococci directly from blood culture bottles in less than 30 minutes. $\mathrm{J}$ Clin Microbiol 2012; 50: 1994-8.

6. Dellinger RP, Levy MM, Rhodes A, et al. Surviving Sepsis Campaign: international guidelines for management of severe sepsis and septic shock, 2012. Intensive Care Med 2013; 39: 165-228.

7. Gaieski DF, Mikkelsen ME, Band RA, et al. Impact of time to antibiotics on survival in patients with severe sepsis or septic shock in whom early goal-directed therapy was initiated in the emergency department. Crit. Care Med 2010; 38: 1045-53.

8. La Scola B, Raoult D. Direct identification of bacteria in positive blood culture bottles by matrix-assisted laser desorption ionisa- 
tion time-of-flight mass spectrometry. PLoS One 2009; 4: e8041.

9. Lane DJ. 16S/23S rRNA sequencing. In: Stackebrandt E, Goodfellow $\mathrm{M}$, eds. Nucleic acid techniques in bacterial systematics. New York: John Wiley and Sons; 1991. pp 115-175.

10. Martin GS, Mannino DM, Eaton S, Moss M. The epidemiology of sepsis in United States from 1979 through 2000. N Engl J Med 2003 ; 348: 1546-54.

11. Samuel LP, Tibbetts RJ, Agotesku A, et al. Evaluation of a microarray-based assay for rapid identification of Gram-positive organisms and resistance markers in positive blood cultures. J Clin Microbiol 2013; 51: 1188-92.
12. Tan KE, Ellis BC, Lee R, et al. Prospective evaluation of a matrixassisted laser desorption ionization-time of flight mass spectrometry system in a hospital clinical microbiology laboratory for identification of bacteria and yeasts: a bench-by-bench study for assessing the impact on time to identification and cost-effectiveness. J Clin Microbiol 2012; 50: 3301-8.

13. Wojewoda CM, Sercia L, Navas M, et al. Evaluation of the Verigene Gram-positive blood culture nucleic acid test for rapid detection of bacteria and resistance determinants. J Clin Microbiol 2013; 51: 20726. 